\title{
Signatures of multiple time-scale behaviour in the power spectra of water
}

\author{
Anirban Mudi ${ }^{\text {a }}$, Ramakrishna Ramaswamy ${ }^{\text {b }}$, Charusita Chakravarty ${ }^{\text {a }}$, \\ a Department of Chemistry, Indian Institute of Technology - Delhi, Hauz Khas, New Delhi 110016, India \\ ${ }^{\mathrm{b}}$ School of Physical Sciences, Jawaharlal Nehru University, New Delhi 110067, India \\ Received 13 May 2003; in final form 13 May 2003 \\ Published online: 12 July 2003
}

\begin{abstract}
Power spectra associated with fluctuations in the tagged particle potential and kinetic energies are analysed for bulk SPC/E water for a range of temperatures along the $1.0 \mathrm{~g} / \mathrm{cm}^{3}$ isochore. Fluctuations in the tagged particle potential energies give rise to $1=\mathrm{f}^{\mathrm{a}}$ noise, indicative of multiple time-scale behaviour, over a temperature-dependent frequency regime. In contrast, the tagged particle centre-of-mass and rotational kinetic energies, which are indicative of the magnitude of local thermal fluctuations, do not show any evidence of $1=\mathrm{f}^{\mathrm{a}}$ behaviour.
\end{abstract}

\section{Introduction}

Structural and dynamical properties of bulk phases of water are qualitatively different from those of simple liquids such as argon, due to the presence of a three-dimensional network of hydrogen bonds [1]. In the liquid phase, the hydrogen-bonded network is dynamic and undergoes structural rearrangements on time-scales comparable to those of molecular rotational and vibrational modes. These hydrogen bond network rearrangements can take place on multiple length and time scales, ranging from fast, localised atomic displacements to large-scale, relatively slow, collective rearrangements. Systems characterised by dynamical processes on multiple time-scales are expected to give rise to a stretched exponential behaviour in the time-correlation functions, $C \partial t P$, with $C \partial t P=\mathrm{C}(0) \exp \left[-(; / \mathrm{x})^{/ \mathrm{J}}\right]$ and a $1=\mathrm{f}^{\mathrm{a}}$-type dependence on the frequency $\mathrm{f}$ in the corresponding power spectrum, $S \partial f P[2,3]$. Multiple time-scales in complex systems can arise from a number of causes. One set of explanations is based on a static picture, where the different time-scales are associated with barrier crossing motions corresponding to a distribution of activation energies [4-6]. Alternative schemes, based on ideas of self-organised criticality, can also give rise to $1=\mathrm{f}^{\mathrm{a}}$-type behaviour [7].

A number of different experimental techniques have been used to explore $1=\mathrm{f}^{\mathrm{a}}$ behaviour of bulk 
liquid water, primarily inelastic neutron scattering, dielectric relaxation measurements and Raman spectroscopy [8]. The background intensity of the Raman spectrum of liquid water in the frequency range $20-4500 \mathrm{~cm}^{11}$ was fitted to a $1=\mathrm{f}^{\mathrm{a}}$ form with $\mathrm{a} \ll 1.3$ [9]. The dielectric relaxation measurements, however, yield single exponential or Debye type relaxation behaviour [10]. Incoherent inelastic neutron scattering experiments yield single particle correlation functions which are consistent with the stretched exponential behaviour [11,12]. More recently, coherent neutron scattering experiments have reported that the resulting time correlation functions can also be fitted to a stretched exponential relaxation form [13]. Molecular dynamics simulations corroborate the experimental finding that, in the case of liquid water, signatures of multiple time scale behaviour are seen for certain experimental quantities, such as the intermediate scattering function but not for others, such as the dielectric relaxation functions. Interestingly, MD simulations can also be performed for finite water clusters and show that the existence of a free surface is sufficient to result in $1=f^{\mathrm{a}}$ behaviour in the power spectra of all the above-mentioned dynamical quantities, including dielectric relaxation $[14,15]$.

In addition to time-correlation functions that correspond to observables of scattering and relaxation experiments, MD simulations allow for the monitoring of dynamical quantities that are inaccessible experimentally. This can be used to understand the microscopic origin of the $1=\mathrm{f}^{\mathrm{a}}$ behaviour in liquid water. Given any mechanical quantity $A \partial t P$ as a function of time $t$ along a molecular dynamics trajectory in the microcanonical ensemble, one can define the power spectrum of the fluctuations about the mean, $h A i$, as

$$
\boldsymbol{S}(\boldsymbol{f})=\left|\int_{t_{\text {min }}}^{t_{\text {max }}}(A(t)-(A)) e^{2 m f l} d t\right|^{2} .
$$

In a previous study, power spectra corresponding to fluctuations in the total, tagged particle and inherent structure potential energies were calculated for a TIPS 2 model of liquid water at $298 \mathrm{~K}$ [7]. Fluctuations in the total and inherent structure energies were found to show $1=\mathrm{f}^{\mathrm{a}}$ behaviour over a three decade range in frequency, with the exponents of 0.75 and 1.3, respectively. Subsequently, fluctuations in a local structure index for water were also found to give rise $1=\mathrm{f}^{\mathrm{a}}$ behaviour [16].

In the present work, we study the $1=\mathrm{f}^{\mathrm{a}}$ behaviour of power spectra for SPC/E water. The extended simple point charge (SPC/E) has been extensively studied and the density, diffusional and compressibility anomalies are well characterised [17-23]. We consider power spectra associated with fluctuations in the tagged particle kinetic, as well as potential, energies. The tagged particle energy is sensitive to changes in the local configurational environment of individual particles. The tagged particle kinetic energy can be thought of as a local temperature measure or as a measure of the strength of local thermal fluctuations. The centre of-mass and rotational contributions to the kinetic energy are monitored separately. The temperature dependence of $1=\mathrm{f}^{\mathrm{a}}$ noise is studied along an isochore in the region of the density anomaly. The computational details are given in Section 2 and the results are discussed in Section 3. Section 4 summarises our conclusions.

\section{Computational details}

The SPC/E model is an effective pair-additive potential energy surface for water which assumes rigid, non-polarizable, three-site model for each molecule [17]. The three sites are located at the atomic positions and are associated with the corresponding atomic masses. The $\mathrm{OH}$ distance is $1.0 \AA$ and $\mathrm{HOH}$ angle is equal to $109.47^{\circ}$, with partial charges on oxygen and hydrogen of $-0.8476 \mathrm{e}$ and $\mathrm{p} 0.4238 \mathrm{e}$, respectively. The Lennard-Jones site is located at the oxygen atom and the corresponding parameters are $r=3.166 \AA$ and $\mathrm{e}=0.6517 \mathrm{~kJ} / \mathrm{mol}$.

The molecular dynamics (MD) simulations were performed using the DL_POLY software package [24]. A cubic simulation cell containing $256 \mathrm{SPC} / \mathrm{E}$ water molecules was used. Electrostatic interactions were evaluated using the Ewald sum approach. The MD trajectory was propagated in the microcanonical (NVE) ensemble using the Verlet leapfrog algorithm in conjunction with the 
SHAKE algorithm to implement bond constraints $[25,26]$. In keeping with earlier work, a time step of 1 fs was used for all the simulations; this resulted in a $0.056 \%$ drift in total energy during the course of a 0.5 ns run.

The equation of state of SPC/E water has been mapped out in previous work. The melting point at $1 \mathrm{~atm}$ pressure is known to be below $260 \mathrm{~K}$ [22]. The minimum in the pressure-temperature, $P \partial T P$, curve has been located at $250 \mathrm{~K}$ along the $1.0 \mathrm{~g} /$ $\mathrm{cm}^{3}$ isochore. We have performed simulations at four temperatures along this isochore spanning the region around the anomalous minimum in the equation of state. At each temperature, the system was equilibrated for a time period $\mathrm{s}_{\mathrm{eq}}$ and data were collected for production runs of length $s_{\text {pro }} d$. Error bars were estimated by block averaging [27]. The key simulation results for the four state points are summarised in Table 1. Within the statistical error bars, our simulation results match those reported for the $P \partial T P$ curves in $[19,20]$; however, our production run lengths at the lowest temperature $(\ll 212 \mathrm{~K})$ are much smaller than those used in previous work. There is a difference of approximately $1 \%$ between the ensemble-averaged potential energy reported in this work and in [19] which may be attributed to the use of Ewald summation, rather than a reaction field method, to account for long-range interactions [22].

In order to compare with the earlier work on $1=f^{a}$ noise in TIPS2 water [7], we computed the power spectrum associated with fluctuations in the total potential energy. The total potential energy is an extensive quantity and the associated fluctuations decrease with increasing system size. Therefore, we preferred to focus on calculation of the power spectrum for the following quantities: (i) the tagged particle potential energy, $u ð t P$, which cor- responds to the interaction energy of an individual molecule with all the other molecules in the system. Since the potential energy surface is assumed to be pair-additive, the total potential energy, $U \partial t P=0: 5$ P $u i ð t P$ where the sum extends over all molecules; (ii) the tagged particle centre-of-mass kinetic energy, $k_{c m} \partial t P$, corresponding to the translational kinetic energy of an individual molecule and (iii) the tagged particle rotational kinetic energy, $k_{r} o t \partial t P$. Since a rigid monomer approximation is made, there is no intramolecular vibrational energy to account for. For 32 molecules in each simulation, the tagged particle potential energies were stored at intervals of $1 \mathrm{fs}$ whereas the tagged particle kinetic energies were sampled at intervals of $10 \mathrm{fs}$. Thus, for each of the 32 tagged molecules, the discrete sampling of a time signal, $A ð t P$, was obtained where $A \partial t P=u \partial t P, k_{c m} \partial t P$ or $k r_{o t}$ and Fourier transformed to generate the corresponding power spectrum, denoted by $S_{u} \partial f P, \mathrm{~S}_{\mathrm{cm}} \partial \mathrm{fP}$ or SrotðfP. Since standard fast Fourier transform (FFT) routines were used, data lengths corresponding to integer powers of two were used [28]. For a time signal represented by $\mathrm{N}$ points, the power spectrum was normalised to ensure that the sum over the $N=2 b 1$ positive frequencies corresponded to the mean square amplitude of the time signal. The most effective method to reduce the signal-to-noise ratio in the power spectrum was found to be averaging over the individual tagged particle spectra. The use of windowing functions did not substantially reduce the noise in the power spectra; therefore all the power spectra reported here correspond to the square window case. In some cases, specially for the total potential energy fluctuations, partitioning the original signal into $ð K b 1 \mathrm{P}$ overlapping segments of equal length and then averaging over power spectra from individual

Table 1

Equation of state data from microcanonical ensemble simulations of SPC/E water at four state points along the $1 \mathrm{~g} / \mathrm{cm}^{3}$ isochore

\begin{tabular}{lllll}
\hline$T$ & $P$ & $U$ & $\begin{array}{l}\mathrm{s}_{\mathrm{eq}} \\
(\mathrm{ns})\end{array}$ & $\begin{array}{c}\text { sprod } \\
(\mathrm{ns})\end{array}$ \\
\hline 212.6 & $(\mathrm{MPa})$ & $(\mathrm{kJ} / \mathrm{mol})$ & 5.0 & 5.0 \\
$253.4 \pm 0.6$ & ) 4.7 & ) 52.58 & 1.0 & 1.0 \\
$292.8 \pm 0.4$ & $-24.1 \pm 1.5$ & $-49.6 \pm 0.1$ & 0.5 & 1.0 \\
$388.4 \pm 0.3$ & $-1.26 \pm 1.2$ & ) $47.1 \pm 0.1$ & 0.5 & 1.0 \\
\hline
\end{tabular}

Error bars obtained using block averaging are not reported for lowest temperature run were the system dynamics is very slow. 
segments was performed in order to reduce the noise. However, in cases where the long-time, low frequency behaviour was of interest, partitioning into only two segments was done.

\section{Results and discussion}

Fig. 1 compares the power spectra associated with the total potential energy fluctuations for the four statepoints listed in Table 1. Comparison with the results for TIPS2 water given in [7] show that the overall shape and qualitative features of the power spectra at different temperatures are very similar for SPC/E and TIPS2 water. For example, in Fig. 1, the $1=\mathrm{f}^{\mathrm{a}}$ region can be seen for the power spectrum between 40 and $300 \mathrm{~cm}^{\prime 1}$. The slopes at lower temperatures vary quite strongly with the frequency range; therefore we have not attempted to do a detailed analysis of the $1=f^{\mathrm{a}}$ behaviour for the total potential energy fluctuations. In the case of TIPS2 water, the power spectrum associated with the total potential energy fluctuations was found to show $1=\mathrm{f}^{\mathrm{a}}$ behaviour with a $\ll 0.75$ and the slope was found to vary appreciably with temperature. However, the frequency range over which the fitting was done at different temperatures is not indicated in the earlier work [7].

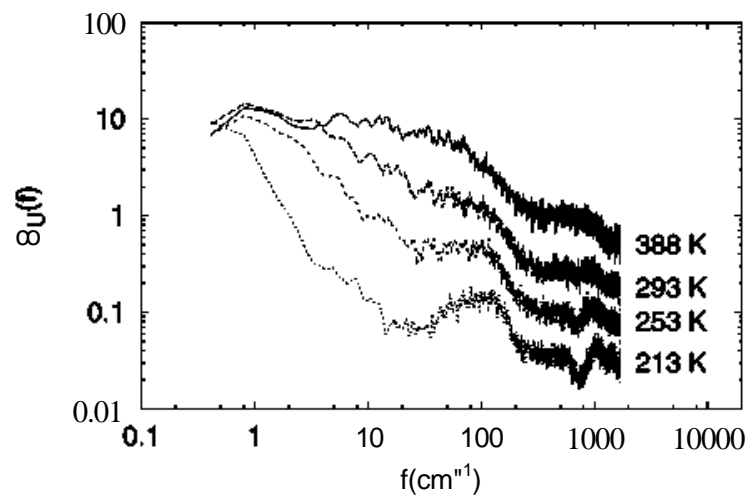

Fig. 1. Power spectra of the total potential energy fluctuations at different temperatures along the $1 \mathrm{~g} / \mathrm{cm}^{3}$ isochore. The length of the time signal was fs $80 \mathrm{ps}$ and the sampling interval was 10 fs corresponding to a Nyquist frequency of $1666 \mathrm{~cm}^{\prime 1}$. Averaging was done over 90 windows.
Fig. 2 compares the $S_{u} \partial f P, S r_{o t} \partial f P$ and $S_{c m} \partial f P$ curves for SPC/E water at a temperature of $293 \mathrm{~K}$ and density of $1 \mathrm{~g} / \mathrm{cm}^{3}$. We first consider the power spectrum associated with fluctuations in the tagged particle potential energies. This quantity has been studied in [7] for TIPS2 water at $290 \mathrm{~K}$. The $S_{u}$ ðfP curve drops fairly rapidly to zero above $1000 \mathrm{~cm}^{\prime 1}$ since intermolecular vibrational modes are absent above this frequency. Between 200 and $1000 \mathrm{~cm}^{\prime 1}$, there is a broad maximum centred at $450 \mathrm{~cm}^{\prime 1}$ which is conspicuous on the logarithmic plot. The librational modes in water are known to lie in the $400-1000 \mathrm{~cm}^{\prime 1}$ frequency regime from experiment as well as simulations. A clear $1=\mathrm{f}^{\mathrm{a}}$ regime can be seen in the frequency interval from 2 to $200 \mathrm{~cm}^{\prime 1}$. The crossover to white noise behaviour occurs at about $1 \mathrm{~cm}^{\prime 1}$. The exponent a in the $1=\mathrm{f}^{\mathrm{a}}$ region was found to be «1.44. The variation in the computational estimate of a with respect to sampling interval as well as length of the time signal was studied in detail. The convergence tests are summarised in Table 2 and indicate that the variation in a is less than 0.06. In the case of TIPS2 water, the tagged particle potential energy fluctuations were found to result in a power spectrum in

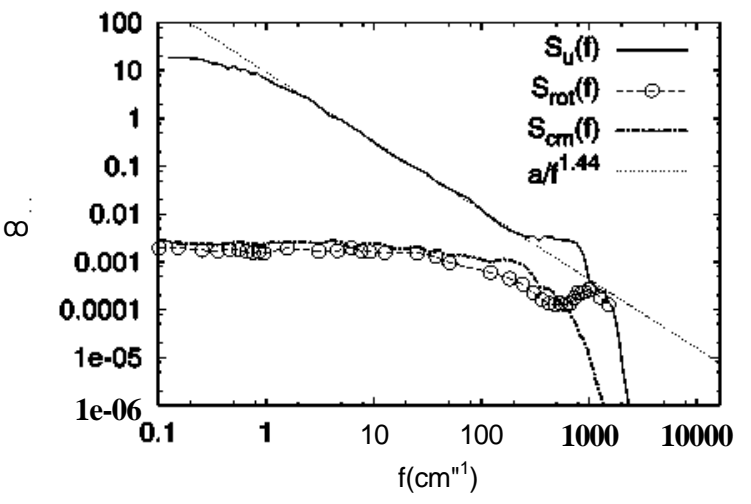

Fig. 2. Comparison of power spectra obtained from fluctuations in the tagged molecule potential energy, rotational and centre-of-mass kinetic energy at $292.8 \pm 0.4 \mathrm{~K}$ and $1 \mathrm{~g} / \mathrm{cm}^{3}$. The length of the time signal for the $S_{u} \partial f p$ curve was $2^{19}$ fs (fsO.5 ns) and the sampling interval was 1 fs corresponding to a Nyquist frequency of $16666 \mathrm{~cm}^{\prime 1}$. The length of the time signal for the $S_{\text {rot }} \partial f P$ and $S c m ð f P$ curves was $2^{16} \times 10$ fs (fsO.65 ns) and the sampling interval was 10 fs corresponding to a Nyquist frequency of $1666 \mathrm{~cm}^{\prime 1}$. For clarity of presentation, logarithmically spaced points are shown on the graph. 
Table 2

Dependence of the exponent in the $1=\mathrm{f}^{\mathrm{a}}$ fit to the power spectrum, $S_{u} \partial f P$, in the frequency range from 2.7 to $245 \mathrm{~cm}^{\prime 1}$ on computational parameters associated with the Fourier transformation of the time signal of the tagged particle potential energy, $u ð t P$

\begin{tabular}{ccc} 
DS (fS) & Data length, $N$ & $a$ \\
1 & 65536 & 1.49 \\
1 & 131072 & 1.43 \\
1 & 262144 & 1.44 \\
1 & 524288 & 1.44 \\
7 & 65536 & 1.45 \\
15 & 65536 & 1.43 \\
\hline
\end{tabular}

The sampling interval, DS, corresponds to the interval between two stored values of $u \partial t \mathrm{P}$ while the data length correspond to the number of sampled values of $u \partial t P$ used as input for the FFT routines. The $u \partial t P$ data were from the simulation at 292.8 $\mathrm{K}$. A square window was used in all cases.

which the intermediate frequency regime could not be well fitted with a straight line though the overall shape and features of the $S_{u} \partial f P$ curve were very similar to those shown for SPC/E water in this work. The differences between TIPS2 and SPC/E water may be attributed to the different potential energy parametrisations and consequent variations in the hydrogen network structure and dynamics.

We now compare $S_{u} \partial f P$ with $S_{c m} \partial f P$ and $S_{\text {rot }} \partial f P$. The fluctuations in the tagged particle kinetic energies are much smaller than those in the potential energy resulting in noisier power spectra and lower values of $S c m ð^{f} P$ and $S_{\text {rot }} \partial^{f} P$ compared to $S_{u} \partial f P$. The $S_{c m} \partial^{f} \boldsymbol{P}$ curve, associated with fluctuations in the tagged particle translational kinetic energy, is essentially a white noise spectrum for $\mathrm{f}<100$ $\mathrm{cm}^{\prime 1}$. Above $100 \mathrm{~cm}^{\prime 1}$, the power spectrum drops fairly rapidly with increasing frequency. This is the type of power spectrum seen for tagged particle potential energy fluctuations in liquid argon. The tagged particle translational kinetic energy fluctuations therefore show no signatures of multiple time-scale behaviour. The corresponding quantity associated with the tagged particle rotational kinetic energy, $S r_{o t} \partial f p$, also shows white noise behaviour below $100 \mathrm{~cm}^{11}$. However, unlike $S_{c m} \partial f P$, SrotðfD is significant for frequencies between 500 and $1500 \mathrm{~cm}^{11}$. A broad peak in $S_{\text {rot }} \partial f b$ centred at about $1100 \mathrm{~cm}^{11}$ occurs in this region. The minimum in $S_{\text {rot }} \partial f P$ at $500 \mathrm{~cm}^{11}$ coincides with the position of the maximum in $S_{u} \partial f P$. This contrast in the high frequency or short-time behaviour is reflected in the behaviour of corresponding autocorrelation functions which are not shown here. While it is obvious that these high frequency features must be related to the hindered rotations of the $\mathrm{H}_{2} \mathrm{O}$ molecules, we do not at present have a molecular level explanation for the locations of the peaks in $S_{u} \partial f P$ and $S_{r o t} \partial f P$.

The $S_{u} \partial f P$ power spectrum at four different temperatures along the $1.0 \mathrm{~g} / \mathrm{cm}^{3}$ isochore is shown in Fig. 3. The $S_{u} \partial f b$ curve at $293 \mathrm{~K}$, discussed above, may be compared with the curve at $388 \mathrm{~K}$. The crossover frequency at $388 \mathrm{~K}$ is « $4 \mathrm{~cm}^{\prime 1}$, compared to $1 \mathrm{~cm}^{\prime 1}$ at $293 \mathrm{~K}$. The high frequency peak centred at $450 \mathrm{~cm}^{11}$ is less pronounced at the higher temperature. The region between 4 and $200 \mathrm{~cm}^{11}$ can be fitted to a $1=\mathrm{f}^{\mathrm{a}}$ form with a «1:43. The $S_{u} \partial f$ p curve at $253 \mathrm{~K}$ has the following significant features: (i) crossover frequency at approximately $0.4 \mathrm{~cm}^{11}$; (ii) pronounced high frequency peak at $450 \mathrm{~cm}^{11}$; (iii) $1=\mathrm{f}^{\mathrm{a}}$ regime between 1 and $200 \mathrm{~cm}^{1}$ with a « 1:38. For the lowest temperature power spectrum at $213 \mathrm{~K}$, no crossover to white noise behaviour can be seen above $0.1 \mathrm{~cm}^{\prime 1}$. In contrast to the spectra at $253 \mathrm{~K}$ and above, $\ln S_{u} \partial f P$ at $213 \mathrm{~K}$ shows a clear non-linear dependence on $\operatorname{lnf}$ between 10 and $200 \mathrm{~cm}^{11}$. Between 0.2 and $10 \mathrm{~cm}^{11}$, one can fit $S_{u} \partial f P$ to a $1=\mathrm{f}^{\mathrm{a}}$ form with a $=1: 47$ but

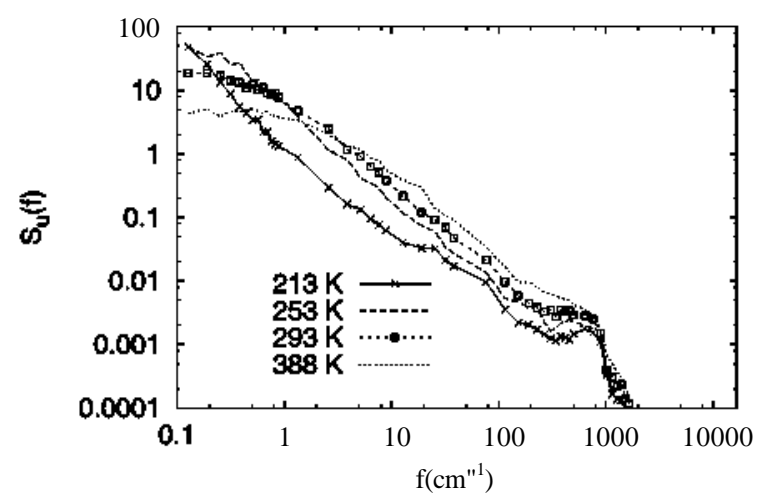

Fig. 3. Power spectra of the tagged molecule potential energies at different temperatures along the $1 \mathrm{~g} / \mathrm{cm}^{3}$ isochore. The computational details are the same as for the $S_{u} \partial f \boldsymbol{b}$ curve in Fig. 2. 
this is a very small frequency domain when compared to the regime for multiple time-scale behaviour at higher temperatures. Since structural relaxation is very slow at such low temperatures, longer runs may be necessary to refine results at the low frequency end of the power spectrum and to locate a crossover frequency. It should also be noted that in the multiple time-scale frequency regime, an effort was made to fit the $S_{u} \partial f D$ curves to a Gaussian-logarithmic form but this did not prove to be significantly better than the simple l/f function [9].

Fig. 4 compares $S_{\text {rot }} \partial f D$ at four different temperatures. Unlike the $S_{u} \partial f P$ curves, the overall shape of

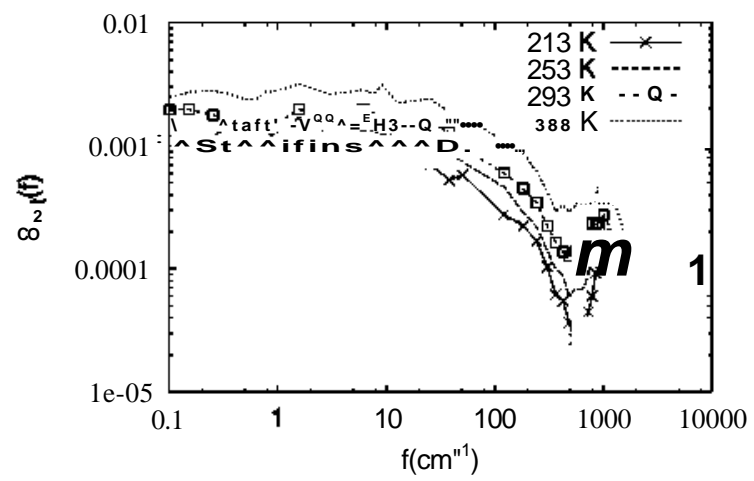

Fig. 4. Power spectra of the tagged molecule rotational kinetic energy at different temperatures along the $1 \mathrm{~g} / \mathrm{cm}^{3}$ isochore. The computational details are the same as for the $S_{\text {rot }} \partial f P$ curve in Fig. 2.

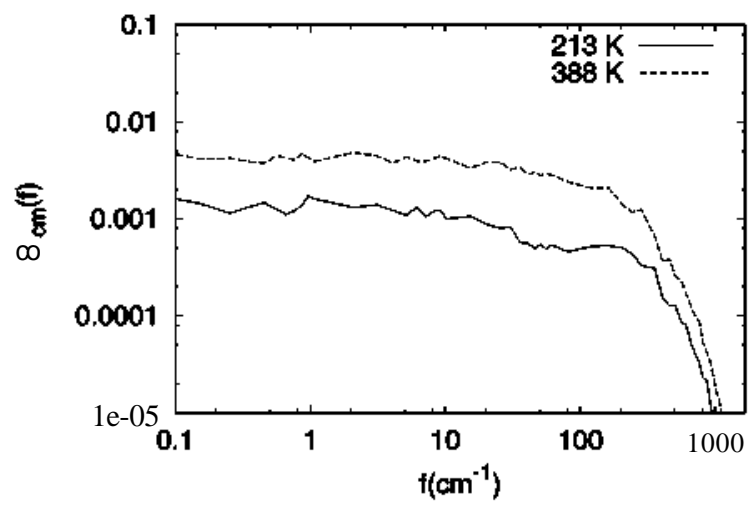

Fig. 5. Power spectra of the tagged molecule translational kinetic energy at different temperatures along the $1 \mathrm{~g} / \mathrm{cm}^{3}$ isochore. The computational details are the same as for the $S_{c m} \partial f P$ curve in Fig. 2. the $S_{\text {rot }} \partial f b$ curves remains the essentially the same with temperature except that the high frequency peak, centred at approximately $1100 \mathrm{~cm}^{11}$, becomes more pronounced at lower temperatures. The ScmðfP curves at four different temperatures, shown in Fig. 5, are all white noise power spectra with a sharp fall in frequency at about $100 \mathrm{~cm}^{\prime 1}$.

\section{Conclusions}

The present work examines in detail the power spectrum, $S_{u} \partial f P$, associated with fluctuations in tagged particle potential energies for SPC/E water over a temperature range spanning the density anomaly along the $1 \mathrm{~g} / \mathrm{cm}^{3}$ isochore. The $S_{u} \partial f P$ curves at temperatures of $253 \mathrm{~K}$ and above have three distinct regions: (i) a low frequency white noise regime below a crossover frequency, $f_{c}$; (ii) a $1=\mathrm{f}^{\mathrm{a}}$ regime for intermediate frequencies and (iii) a high-frequency region with localised peaks due to restricted rotational modes. The crossover frequency, $f_{c}$, is shown to increase with temperature. The high-frequency peak associated with librational motion becomes less pronounced with temperature. The frequency range associated with $1=\mathrm{f}^{\mathrm{a}}$ or multiple time-scale behaviour is shown to be temperature dependent. For temperatures corresponding to strong supercooling, the power spectra of the tagged particle potential energies is qualitatively different.

Power spectra associated with tagged particle kinetic energies are also studied along the isochore. The fluctuations in the tagged particle centre-ofmass kinetic energies appear to be completely uncorrelated and give rise to a white-noise spectrum with a frequency cut-off at $100 \mathrm{~cm}^{11}$. The fluctuations in the tagged particle rotational kinetic energies show no long time-scale correlations; however, a pronounced peak is seen at high-frequencies which must be related to the restricted rotational motion of the water molecules.

An interesting outcome of this study is that different tagged particle quantities can show very different sensitivities to the multiple time-scale relaxations of the hydrogen-bonded network of water. The tagged particle potential energies show a $1=\mathrm{f}^{\mathrm{a}}$ regime for temperatures above and close to the 
freezing temperature. In complete contrast, the fluctuations in the tagged particle translational or centre-of-mass kinetic energies are completely of the Markovian or white-noise type. This is consistent with a structural origin for the multiple time-scale behaviour and also suggests that it may be worth exploring which other structural quantities give rise to $1=\mathrm{f}^{\mathrm{a}}$-type behaviour in order to gain further insights into the multiple time-scale behaviour.

\section{Acknowledgements}

Financial support for this work has been provided by the Council for Scientific and Industrial Research (01 (1636)/EMR-II) and the Department of Science and Technology (SP/S1/H-16/2000). CC and AM thank the Computer Services Centre of I.I.T. - Delhi for access to their computational resources. AM would like to thank the Council for Scientific and Industrial Research, New Delhi for the award of a Junior Research Fellowship.

\section{References}

[1] F. Franks (Ed.), Water: A Comprehensive Treatise, Plenum Press, New York, 1972.

[2] D.A. McQuarrie, Statistical Mechanics, University Science Books, 2000.

[3] P.M. Chaikin, T.C. Lubensky, Principles of Condensed Matter Physics, Cambridge University Press, Cambridge, MA, 1995.

[4] F. Sciortino, P.H. Poole, H.E. Stanley, S. Havlin, Phys. Rev. Lett. 64 (1990) 1686.

[5] S.K. Nayak, R. Ramaswamy, C. Chakravarty, Phys. Rev. Lett. 74(1995)4181.
[6] S.K. Nayak, R. Ramaswamy, Surf. Rev. Lett. 3 (1996) 457.

[7] M. Sasai, I. Ohmine, R. Ramaswamy, J. Chem. Phys. 96 (1992) 3045.

[8] I. Ohmine, J. Phys. Chem. 99 (1995) 6767.

[9] G.E. Walrafen, M.S. Hokmabadi, W.-H. Yang, Y.C. Chu, B. Monosmith, J. Phys. Chem. 93 (1989) 2909.

[10] D. Bertollini, M. Cassettrari, M. Ferrario, P. Grigolini, G. Salvetti, Adv. Chem. Phys. 62 (1985) 277.

[11] P. Gallo, F. Sciortino, P. Tartaglia, S.-H. Chen, Phys. Rev. Lett. 76 (1996) 2730.

[12] S.-H. Chen, C. Liao, F. Sciortino, P. Gallo, P. Tartaglia, Phys. Rev. E. 59 (1999) 6708.

[13] M.C. Bellisent-Funel, S. Longeville, J.M. Zanotti, S.H. Chen, Phys. Rev. Lett. 85 (2000) 3644.

[14] S. Saito, I. Ohmine, J. Chem. Phys. 102 (1995) 3566.

[15] A. Baba, Y. Hirata, S. Saito, I. Ohmine, D.J. Wales, J. Chem. Phys. 106 (1997) 3329.

[16] E. Shiratani, M. Sasai, J. Chem. Phys. 104 (1996) 7671.

[17] H.J.C. Berendsen, J.R. Grigera, T.P. Straatsma, J. Phys. Chem. 91 (1987) 6269.

[18] K. Bagchi, S. Balasubramanian, M.L. Klein, J. Chem. Phys. 107 (1997) 8561.

[19] F.W. Starr, F. Sciortino, H.E. Stanley, Phys. Rev. E. 60 (1999) 6757.

[20] S. Harrington, P.H. Poole, F. Sciortino, H.E. Stanley, J. Chem. Phys. 107 (1997) 7443.

[21] P.A. Netz, F.W. Starr, H.E. Stanley, M.C. Barbosa, J. Chem. Phys. 115 (2001) 344.

[22] S.C. Gay, E.J. Smith, A.D.J. Haymet, J. Chem. Phys. 116 (2002) 8876.

[23] M.E. Parker, D.M. Heyes, J. Chem. Phys. 108 (1998) 9039.

[24] W. Smith, T.R. Forester, J. Mol. Graphics 14 (1996) 136.

[25] M.P. Allen, D.J. Tildesley, Computer Simulation of Liquids, Oxford University Press, Oxford, 1986.

[26] J.P. Ryckaert, G. Ciccotti, H.J.C. Berendsen, J. Comput. Phys. 23 (1977) 327.

[27] H. Flyvberg, H.G. Petersen, J. Chem. Phys. 91 (1989) 461.

[28] W.H. Press, B.P. Flannery, S.A. Teukolsky, W.T. Vetterling, Numerical Recipes in Fortran, Cambridge University Press, Cambridge, MA, 1990. 